\title{
Short- and long-term cognitive effects of deep brain stimulation in the caudal zona incerta versus best medical treatment in patients with Parkinson's disease
}

\author{
Johanna Philipson, MS, ${ }^{1}$ Patric Blomstedt, PhD, ${ }^{1}$ Anna Fredricks, ${ }^{1}$ Marwan Hariz, MD, PhD, ${ }^{1,2}$ \\ Rasmus Stenmark Persson, MD, ${ }^{1}$ and Marjan Jahanshahi, PhD2,3
}

1Department of Pharmacology and Clinical Neuroscience, Umeå University, Umeå, Sweden; 2Unit of Functional Neurosurgery, UCL Institute of Neurology, London, United Kingdom; and ${ }^{3}$ The Clinical Hospital of Chengdu Brain Science Institute, MOE Key Lab for Neuroinformation, University of Electronic Science and Technology of China, Chengdu, China

OBJECTIVE A growing number of studies are showing positive effects of deep brain stimulation (DBS) in the caudal zona incerta (CZi) in various tremor disorders, as well as motor symptoms of Parkinson's disease (PD). The focus of the present study was to evaluate short- and long-term cognitive effects of bilateral cZi DBS in patients with PD.

METHODS Twenty-five nondemented patients with advanced PD were recruited to participate in a randomized trial of cZi DBS versus best medical treatment (BMT). The patients in the BMT group were offered surgery after 6 months. Neuropsychological evaluations focusing on assessing verbal and visuospatial memory, attention, and executive function were conducted at baseline and at 6 and 24 months after surgery. Self-reported measures of depression, anxiety, and change in "frontal" behaviors were also completed at all assessment points.

RESULTS Bilateral cZi DBS in patients with PD generated few adverse cognitive effects. At the short-term follow-up after 6 months, no differences were found between patients randomized to BMT and patients randomized to DBS with regard to most of the cognitive domains assessed. A transient improvement in anxiety was, however, found in the surgical group. At the long-term follow-up 24 months after cZi DBS, no major changes in global cognitive functioning were found, although a decline in attention and self-reported executive function was noted.

CONCLUSIONS With the exception of a decline in attention and self-reported executive function, bilateral cZi DBS for PD in appropriately screened patients appears to be generally safe with regard to cognitive function, both in the shortand long-term perspective.

https://thejns.org/doi/abs/10.3171/2019.12.JNS192654

KEYWORDS zona incerta; Parkinson's disease; deep brain stimulation; neuropsychology; cognition; functional neurosurgery

$\mathrm{T}$ HE subthalamic nucleus (STN) is presently the preferred target for deep brain stimulation (DBS) treatment of motor symptoms of Parkinson's disease (PD) when complications of dopaminergic therapy develop. ${ }^{12,41}$ Alternative targets, such as the globus pallidus internus (GPi) or the ventral intermediate nucleus of the thalamus (Vim) may be preferred depending on the symptomatic profile or due to other patient-specific considerations. ${ }^{16,31}$ In recent years, DBS using the posterior subthalamic area (PSA) as a primary target has become increasingly common. This area was frequently used during the lesional era. ${ }^{2,14,22,29,37}$ The PSA is located ventral to the Vim, between the red nucleus and the STN. Among other structures and pathways, the PSA also includes the fields of Forel, the lemniscus, the prelemniscal radiations, and the caudal zona incerta (cZi). ${ }^{6,11}$

Apart from motor symptoms of PD, nonmotor symptoms are also a common feature of PD. Among the nonmotor symptoms, mild cognitive impairment (MCI) has been shown to be present even in early stages of the disease.

ABBREVIATIONS BAI = Beck Anxiety Inventory; BDI = Beck Depression Inventory; BMT = best medical treatment; BVMT-R = Brief Visuospatial Memory Test Revised; CWIT = Color Word Interference Test; $\mathrm{CZi}=$ caudal zona incerta; DBS = deep brain stimulation; FrSBe = Frontal Systems Behavioral Rating Scale; $\mathrm{GPi}=$ globus pallidus internus; $\mathrm{MCl}$ = mild cognitive impairment; PASAT = Paced Auditory Serial Addition Test; PD = Parkinson's disease; PDQ-39 = Parkinson's Disease Questionnaire; PSA = posterior subthalamic area; STN = subthalamic nucleus; TMT = Trail Making Test; UPDRS-III = Unified Parkinson's Disease Rating Scale-III; Vim = ventral intermediate nucleus of the thalamus; WCST-64 = Wisconsin Card Sorting Test 64-card version.

SUBMITTED September 27, 2019. ACCEPTED December 10, 2019.

INCLUDE WHEN CITING Published online February 7, 2020; DOI: 10.3171/2019.12.JNS192654. 
Many studies have suggested that MCI is present in 19\%$36 \%$ of recently diagnosed and de novo patients. ${ }^{1,13,15,28,30}$ Impairments include executive dysfunction as well as memory and visuospatial problems. Dementia is usually a feature of the mid to late stage of PD, and over the long term $80 \%$ of patients with PD develop dementia. ${ }^{19,21}$

The cognitive effects of STN DBS in PD have been investigated in several controlled studies ${ }^{36,39,41,42,44}$ and meta-analyses..$^{9,32}$ These have established that STN DBS is relatively safe from a cognitive perspective and does not produce any adverse effects on global aspects of cognition or on most cognitive domains. The main effect of STN DBS on cognition is a decline in semantic and phonemic verbal fluency, which has been consistently found across studies. ${ }^{9,32,43}$ There is also evidence for deficits in inhibition of prepotent responses with STN DBS on cognitive tasks, such as the Stroop interference effect or random number generation. ${ }^{25-27,38}$

Because a growing number of studies have shown positive results of DBS in different parts of the subthalamic area, ${ }^{7,33}$ and the fact that the target possibly lacks the psychiatric side effects that have been seen after STN DBS, we wanted to evaluate the potential efficacy and safety of cZi as a target for DBS in patients with PD. Our primary study ${ }^{7}$ showed a positive effect on motor symptoms, with $47 \%$ better Unified Parkinson's Disease Rating Scale-III (UPDRS-III) motor scores off medication and on stimulation, compared to the best medical treatment (BMT) patients off medication at 6 months. Preliminary results of the 24-month follow-up indicate a $35.7 \%$ improvement of UPDRS-III scores after 24 months $(\mathrm{p}=0.000122)$.

To our knowledge, the impact of cZi DBS on cognitive function in patients with PD has not been previously reported. Therefore, the aim of this study was to evaluate the short- and long-term cognitive effects of bilateral cZi DBS in patients with PD.

\section{Methods}

This study was part of a randomized controlled trial of cZi DBS for patients with advanced PD. ${ }^{7}$ The primary outcome measures for this study were the UPDRS-III and Parkinson's Disease Questionnaire (PDQ-39). Inclusion criteria were: 1) clinical diagnosis of idiopathic PD according to the UK Parkinson's Disease Society Brain Bank criteria; 2) L-dopa responsive symptoms (defined as $>30 \%$ improvement in motor scores of the UPDRS-III; 3) disabling motor fluctuations with or without dyskinesias, or disabling tremor; 4) age $<75$ years; 5) no psychiatric comorbidities or apparent cognitive decline; 6) normal brain MRI; and 7) no surgical contraindications. Besides a neuropsychological evaluation, all patients also underwent a standardized L-dopa challenge. Informed consent was obtained according to the Declaration of Helsinki (2008 revision). The study was approved by the Ethical Committee of the University Hospital of Northern Sweden.

\section{Study Design}

Patients were randomized to either bilateral cZi DBS within 1 month from inclusion or to BMT. Patients in the BMT group were offered surgery after 6 months. Ran- domization was performed manually by a senior neurologist not participating in the selection/inclusion of patients. Neuropsychological assessments were completed at baseline before surgery as well as at 6 and 24 months postoperatively for all patients. The group randomly allocated to undergo delayed surgery in effect had two baseline assessments (Fig. 1).

\section{Neuropsychological Assessment}

At baseline, the neuropsychological assessments were completed with patients on medication. All assessments postoperatively were performed with patients on stimulation and on medication. The neuropsychological tests used are detailed below.

\section{Global Cognitive Function}

Raven's Coloured Progressive Matrices ${ }^{34}$ is a nonverbal measure of intelligence, global mental ability, and nonverbal reasoning, with a maximum score of 36 . Results are presented in percentiles, standardized based on age group.

\section{Memory}

The Claeson-Dahls Test $^{8}$ assesses verbal learning and memory. A list of 10 words (in Swedish) is read aloud. After a 15-second pause, as many words as possible are to be repeated. The test is completed after a maximum of 10 repetitions of the word list, or when the patient correctly recalls all words two consecutive times. After 30 minutes, a recall trial is administered. Results are calculated based on the number of words remembered and the number of times the list of words needs to be repeated. A low score indicates a faster learning curve.

The Brief Visuospatial Memory Test-Revised (BVMT$\mathrm{R})^{5}$ is a test for assessment of visual memory and comprises 6 alternate forms, each consisting of 6 geometrical figures. The figures are presented for 10 seconds. The participant is asked to draw as many of the geometrical figures as possible, preferably matching the stimulus card with regard to placement and accuracy. The figures are presented three times to assess for short-term spatial memory and learning. After 30 minutes delayed recall is tested followed by a delayed recognition task. Scores are given for accuracy and placement and range from 0 to 12 . Higher scores indicate better visual memory.

The Wechsler Adult Intelligence Scale-IV, digit forward and backward, ${ }^{40}$ is a test of short-term verbal memory and verbal working memory. Digit span is measured for forward- and reverse-order (backward) recall of digit sequences. The sequences are initially presented with a length of two digits with two trials at each increasing digit length. Testing stops when the patient reports both trials incorrectly at one sequence length, or when the maximal list length is reached (9 digits forward, 8 backward). Scores range from 0 to 18 forward and 0 to 16 backward, with higher scores indicating better performance. Raw scores are converted to normative scaled scores stratified by age ranging from 1 to 19 , with higher scores indicating better performance.

\section{Executive Function}

The Delis-Kaplan Executive Function System ${ }^{10}$ is a 


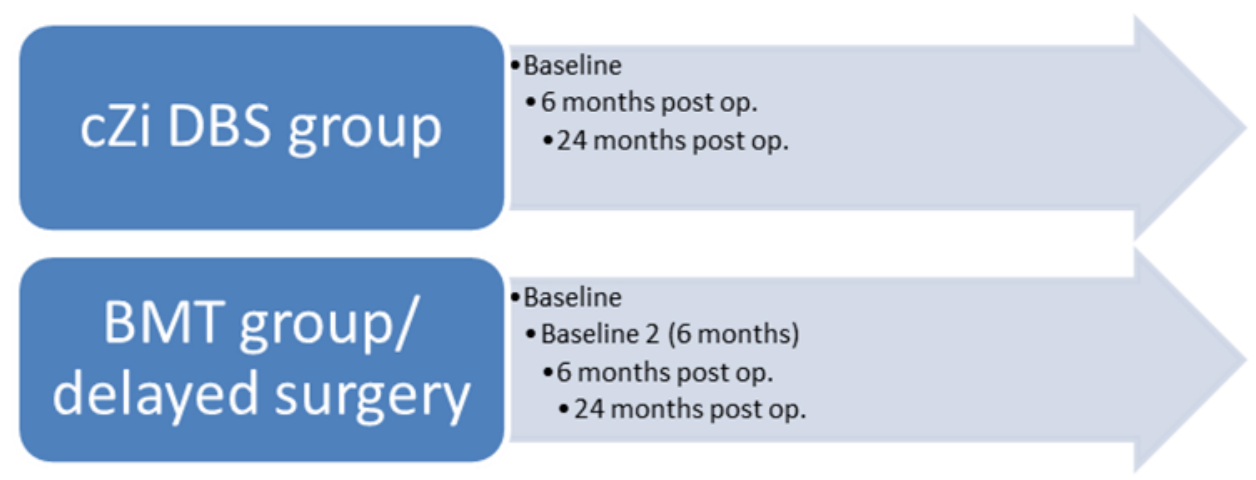

FIG. 1. Illustration showing the two treatment groups (cZi DBS and BMT delayed/surgery) and the time points of neuropsychological assessment. Figure is available in color online only.

standardized test for assessing executive functions and other higher-level cognitive functions.

The Trail Making Test (TMT) is a test of visual attention, behavioral regulation, task switching, and processing speed. The test consists of 5 individual subtests: 1) visual scanning: the patient is asked to mark circles with the number "3" presented among both letters and numbers; 2 ) number sequencing: the patients is asked to connect numbers in successive order; 3 ) letter sequencing: the patient is asked to connect letters in alphabetical order; 4) numberletter switching: the patient is asked to connect circles in an alternating sequence, switching between numbers and letters (1-A-2-B-...); and 5) motor speed: requires the patient to follow a dashed line as quickly as possible. Scores are based on the time needed to complete the individual subtests. Results are transformed to scaled scores ranging from 1 to 19, where a higher score indicates better performance.

Verbal fluency is a test of verbal functioning consisting of 3 tasks: phonemic fluency (sometimes called letter fluency), category fluency (also called semantic fluency), and switching category fluency. One minute is given for the patient to say as many unique words as possible, starting with a given letter (letter fluency) or within a semantic category (category fluency). The score in each task is based on the number of correct words. In the last task, the participant is asked to shift between semantic categories. Raw scores are transformed into scaled scores ranging from 1 to 19 , where a higher score indicates better performance.

The Stroop Color Word Interference Test (CWIT) has 4 subtests: 1) color naming: naming the color of colored rectangles; 2) word reading: reading color words (red, green, blue) printed in black ink; 3) color word interference/inhibition: naming the color of colored words printed in incongruent ink, for example, the word "red" printed in blue ink; the test focuses on the capability to inhibit the more habitual response of reading the words, and instead engage in the more unconventional response of naming the color of ink they are printed in; and 4) inhibition-switching: the same as condition 3 (color word interference/inhibition), except when there is a box around the word, the participant has to read the word. Thus, this condition requires both inhibition of habitual responses and switching response strategy across trials. The total time to complete each of the subtests and the number of self-corrected and uncorrected errors are recorded. Scores are transformed into scaled scores ranging from 1 to 19 , where a higher score indicates a better performance.

The Wisconsin Card Sorting Test 64-card version (WCST-64) ${ }^{20}$ is a test administered only preoperatively and at the 24-month follow-up. A computerized version was used in which 4 stimulus cards incorporate 3 different stimulus parameters (color, shape, and number). The participant is supposed to sort the cards according to the sorting parameters, and to alter their sorting strategy based on the feedback they receive during the test. The test is designed to measure the ability to develop and maintain a correct problem-solving strategy across changing conditions and to alter cognitive strategies in response to changing contingencies. The number of correctly sorted categories and the number/types of errors are recorded. Scores are reported after being transformed into T-values and percentiles based on age and education. T-values range from $<20$ to $>80$, with a higher score indicating better performance.

\section{Attention}

The Dichotic Listening Task ${ }^{23}$ is a test for studying brain asymmetry in auditory processing. The participant is simultaneously presented with different sounds to the right and left ear. Two different kinds of cognitive processes are measured using 3 conditions: 1) a lateralized perceptual process (the nonforced condition); 2) an attention process (the forced-right condition); and 3) an executive cognitive control process (the forced-left condition). The first condition is generally used to determine speech lateralization. The results from the 2 forced conditions are calculated as a percentage correct score of the number of stimuli presented to the right and left ear, respectively. The raw score is transformed to a T-score ranging from $<20$ to $>80$. Interpretation of the T-scores are made with respect to the condition being tested and indicate, for instance, the test subject's ability to shift auditory attention.

The Paced Auditory Serial Addition Test (PASAT) assesses attention, working memory, speed of auditory information processing, as well as calculation skills. Single digits are read out loud every 3 seconds (by use of a pre- 
recorded audio tape). The participant is asked to add each new digit to the one presented directly prior to it and verbally report the sum. The correct answers given constitute the raw score, which is then transformed to percentiles and $\mathrm{Z}$-scores with higher scores indicating better performance.

\section{Assessment of Mood and Behavior}

The Beck Anxiety Inventory (BAI) ${ }^{3}$ and Beck Depression Inventory (BDI) ${ }^{4}$ are each 21 -item self-report measures of anxiety and depression, respectively. The scores range from 0 to 63, with higher scores indicating higher risk of anxiety and depression.

The Frontal Systems Behavioral Rating Scale $(\mathrm{FrSBe})^{18}$ consists of 2 rating forms: a self-report questionnaire and a questionnaire completed by a relative, regarding symptoms or behaviors indicating frontal/prefrontal dysfunction. Each FrSBe form provides a total score and scores for 3 subscales: apathy (14 items), disinhibition (15 items), and executive dysfunction (17 items). Each item is rated on a 5-point Likert sale. Scores are acquired on each subscale for both premorbid and current behavior. T-scores are generated from raw scores using normative tables based on sex, age, and education. T-scores have a mean of 50 and standard deviation of 10 , with higher scores indicating greater pathology.

\section{Study Sample}

Of the 25 patients who initially met the inclusion criteria and agreed to be included and randomized in the trial, 7 patients could not or would not fulfill the trial. Four patients (1 randomized to DBS and 3 to BMT) quit after randomization and did not want to continue. One patient randomized to the DBS group broke her knee after a fall shortly before surgery. Two patients chose to terminate their participation in the study after the surgical procedure was performed. Eighteen patients were thus included at baseline. One additional patient from the BMT group dropped out at the 6-month postoperative follow-up and did not want to participate in any further evaluations, leaving 17 patients at the 6-month follow-up. At the 24-month follow-up, an additional patient chose not to complete the neuropsychological assessment, leaving a total of 16 patients for the last follow-up assessment. The demographic and clinical characteristics of the patients included are presented in Table 1.

\section{Surgical Procedures}

Details of the surgical procedure have been described in a previous publication. ${ }^{7}$ All surgeries were performed by the same surgeon (P.B.). Bilateral stereotactic implantation of the DBS 3389 electrodes (Medtronic) was performed using the Leksell frame model G (Elekta Instruments). The brain target was identified anatomically on stereotactic thin-slice T2-weighted axial MR images. The target point lay slightly posteromedial to the visualized posterior tail of the STN on the scan showing the maximal diameter of the red nucleus. Either an intraoperative stereotactic CT scan or an early postoperative CT scan was performed and fused with the preoperative stereotactic MRI for verification of the electrode position before im-
TABLE 1. Demographic and clinical characteristics of the patients

\begin{tabular}{lcc}
\hline \multicolumn{1}{c}{ Characteristic } & BMT Group & Surgical Group \\
\hline No. of patients at baseline & 9 & 9 \\
\hline Males/females & $7 / 2$ & $7 / 2$ \\
\hline Age at surgery, yrs & $60.3 \pm 8$ & $55.6 \pm 11.3$ \\
\hline Disease duration, yrs & $10.3 \pm 5.6$ & $6.4 \pm 3$ \\
\hline LEDD, mg & & \\
\hline At baseline & $1064 \pm 543$ & $1376 \pm 883$ \\
\hline At 6 mos & $831 \pm 524(n=8)$ & $1054 \pm 488(n=9)$ \\
\hline At 24 mos & $1265 \pm 852(n=8)$ & $1241 \pm 590(n=8)$ \\
\hline UPDRS-III at baseline & & \\
\hline \multicolumn{1}{c}{ Off medication } & $42.4 \pm 14.5$ & $33.2 \pm 11.4$ \\
\hline On medication & $20.6 \pm 11.9$ & $19.4 \pm 12.5$ \\
\hline Yrs of education $(n=18)$ & $10.67 \pm 1.936$ & $10.44 \pm 2.506$ \\
\hline MCl at inclusion $(n=18)$ & $2 / 9$ & $3 / 9$ \\
\hline
\end{tabular}

LEDD = levodopa-equivalent daily doses.

Values represent mean \pm SD unless otherwise indicated. All patients at 24 months had undergone DBS, therefore the LEDD at 24 months in the BMT group concerns patients who had DBS for 24 months, i.e., who belonged to the BMT group initially but had delayed surgery. There were no significant differences between the groups for any variable (all $p$ values nonsignificant).

plantation of the primary cell implantable pulse generator (Medtronic). Microelectrode recording was not used.

After surgery, the electrode contacts were all screened for effects and side effects. The contacts showing best effect, and acceptable side effects, were used for chronic stimulation. Medications and stimulation settings were evaluated and continuously optimized during the study period. All adverse events were also recorded. In the BMT group, patients were monitored according to standard procedures and optimized with regard to current medication by a senior movement disorder neurologist.

The clinical results from the 6-month follow-up have been reported previously ${ }^{7}$ and the 24-month follow-up regarding primary outcomes (UPDRS-III and PDQ-39) will be published in an upcoming report.

\section{Statistical Analyses}

A small percentage of the data was missing for the following reasons: color blindness ( $\mathrm{n}=2$ for both the WCST and CWIT, 1 in each treatment group), problems recording time in the TMT motor speed subtest $(n=1)$, and 2 individuals not completing the BAI (one preoperatively, and one preoperatively, at 6 months, and at 24 months).

For the short-term effects at 6 months, analysis of covariance was used to test for group differences between surgery and BMT controls, with the 6-month follow-up measurement of the corresponding outcome variable as the dependent variable, group as an explanatory factor, while controlling for the baseline measurement as a covariate.

To test for differences between different time points in outcome variables, mixed models were fitted with corresponding outcome variables as dependent variables, time points (preoperative baseline, 6-month follow-up, or 
24-month follow-up) as fixed factors, and subject as random factor. The correlation structure of the mixed models allowed different error variance at each of the 3 time points. For this analysis, the data from the second baseline of the BMT/delayed surgery group were pooled with the baseline of the cZi DBS group, and the delayed surgery group's 6-month postoperative data were pooled with the cZi DBS group's 6-month postoperative data.

All analyses were performed using the $\mathrm{R}$ statistical program (version 3.5.3; https://www.R-project.org/). The mixed models were fitted using the lme function from $\mathrm{R}$ package nlme.

\section{Results}

The effect on motor symptoms after DBS in the cZi have been published elsewhere. ${ }^{7}$ In short, the DBS-treated patients had $47 \%$ better UPDRS-III motor scores off medication and on stimulation compared to the BMT patients off medication at 6 months. DBS patients showed $45 \%$ improvement of UPDRS-III scores off medication and on stimulation, compared to their off-stimulation condition, and $41 \%$ improvement compared to baseline UPDRS-III scores. The final results of the 24-month follow-up have not been published yet but UPDRS-III data show a mean 13.39-point reduction, indicating a $35.7 \%$ improvement after 24 months $(\mathrm{p}=0.000122)$.

\section{Effects of cZi DBS on Cognition}

There were no significant differences in cognition between the surgical group and the BMT group at the preoperative assessment (all $p>0.05$ ). There were also no significant differences between the first and second preoperative assessments of the BMT group or the second preoperative assessment of the BMT and the preoperative assessment of the surgical group (all $\mathrm{p}>0.05$ ).

\section{Short-Term Effects}

Table 2 presents data comparing baseline and 6-month cognitive assessments for the surgical group (cZi DBS) and the BMT group. The only significant difference was an increase of errors in the DBS group on the inhibition subtest of the CWIT. All other measures showed no significant difference over the 6-month period between the two groups.

\section{Long-Term Effects}

The mean and standard deviation of the cognitive measures at baseline and at 6- and 24-month follow-up for the combined cZi DBS patients who had immediate or delayed surgery are presented in Table 3; the $\mathrm{p}$ values for the tests of cognitive function that showed change significant at $\mathrm{p}<0.05$ are highlighted.

As shown in Table 3, a decline in auditory attention, as measured by the PASAT, was found at the 24-month follow-up relative to preoperative baseline. A decline was also noted between the baseline assessment and the 24-month follow-up on one subtest of the Dichotic Listening Task. This subtest requires the patient to report the sound perceived in the left ear, but instead they report back what was presented in the right ear. Another measure showing change over time was the self-report questionnaire probing for executive difficulties (the FrSBe). There was an increase of self-perceived executive dysfunction when comparing the baseline with the 24-month followup. We also found a reduction of anxiety symptoms as measured by the BAI at 6 months. However, this was no longer present at the 24-month follow-up.

\section{Discussion}

This study indicates that bilateral cZi DBS in appropriately screened patients with advanced PD results in very few significant changes in cognitive function. At the 6-month follow-up, the only change we found between the BMT group and the surgical group was a slight increase in the number of errors made during the CWIT inhibition subtest. This indicates that the short-term effect of $\mathrm{cZi}$ DBS on cognition in patients with PD is minor.

When making within-group comparisons over time we found changes primarily in measurements focusing on attention and self-reported executive function. This study thus concludes that there is no reduction in overall cognition. However, a selective decrease in performance on "frontal" cognitive functions relating to attention and executive function was noted. Whether these changes are related to the surgical procedure, electrical stimulation, or merely a reflection of the natural course of cognitive decline in PD, cannot be determined at this time.

Regarding the Dichotic Listening Task, there may be an influence of cZi DBS on aspects of auditory attention worthy of further investigation. The forced-left condition requires a top-down attention process, relying on the ability to voluntarily shift and regulate attention and thus also being closely tied to executive function. We have found similar changes in a previous study ${ }^{35}$ focusing on ear advantage in PD patients who underwent STN DBS. However, impaired hearing is common in the age groups most frequently included for treatment of advanced PD, which makes this type of investigation rather difficult. It is also well known that the Dichotic Listening Task, particularly the forced-left condition, is sensitive to increased age. ${ }^{24}$ It has been suggested that older individuals ( $\geq 60$ years) show decreased performance in the forced-left condition, possibly due to cognitive decline in areas such as cognitive control and executive functioning. ${ }^{24} \mathrm{~A}$ similar conclusion was drawn in a study in which increased asymmetry was found in the focused-attention task in an elderly population. ${ }^{17}$ This, however, was not seen in the nonforced attention condition, thereby suggesting decline in frontal activity in the elderly. The results of the present study may be confounded by subclinical impaired hearing and older age in this sample. No preoperative hearing test was performed and test results were assumed to be valid if the patient claimed to have no impairments regarding hearing and all homonyms were correct within the test.

A recent meta-analysis of the cognitive impact of DBS on PD concluded that STN or GPi DBS produces few cognitive side effects in preoperatively appropriately screened patients. ${ }^{9,32}$ This finding is very consistent with our results, in which few cognitive short- or long-term side effects 
TABLE 2. Short-term effects of cZi DBS on cognition and mood

\begin{tabular}{|c|c|c|c|c|c|c|c|}
\hline \multirow[b]{2}{*}{ Test } & \multicolumn{2}{|c|}{ Surgical Group } & \multicolumn{2}{|c|}{ BMT Group } & \multirow[b]{2}{*}{ Diff } & \multirow[b]{2}{*}{$95 \% \mathrm{Cl}$} & \multirow[b]{2}{*}{$\mathrm{p}$ Value } \\
\hline & Preop & 6 Mos & Preop & 6 Mos & & & \\
\hline \multicolumn{8}{|l|}{ Global cognition } \\
\hline Ravens's Coloured Progressive Matrices (\%) & $48.7(24.8)$ & $65.8(18.9)$ & $71.3(36.0)$ & $69.3(30.6)$ & 13.1 & -10.4 to 36.5 & 0.25 \\
\hline \multicolumn{8}{|l|}{ Memory, T-score } \\
\hline Claeson-Dahls retention & $50.2(7.61)$ & $41.1(13.4)$ & $49.0(8.7)$ & $48.3(5.6)$ & -7.2 & -18.2 to 3.8 & 0.18 \\
\hline Claeson-Dahls verbal learning & $47.0(9)$ & $43.7(5.68)$ & $45.3(7.8)$ & $46.4(9.3)$ & -3.2 & -9.6 to 3.3 & 0.31 \\
\hline BVMT-R recall & $37.8(8.9)$ & $38.8(7.8)$ & $41.1(16.3)$ & $41.9(11.1)$ & -1.8 & -8.8 to 5.3 & 0.60 \\
\hline BVMT-R learning & $54.9(7.9)$ & $55.3(11.5)$ & $52.9(11.19)$ & $54.9(9.9)$ & -0.8 & -12.3 to 10.7 & 0.89 \\
\hline BVMT-R delayed recall & $45.0(9.3)$ & $45.9(7.67)$ & $44.2(13.5)$ & $49.0(10.9)$ & -3.8 & -10.1 to 2.5 & 0.21 \\
\hline \multicolumn{8}{|l|}{ Executive function, scaled score } \\
\hline Phonemic fluency & $10.2(3.8)$ & $9.6(2.8)$ & $11.0(2.7)$ & $11.4(2.4)$ & -1.3 & -3.8 to 1.1 & 0.27 \\
\hline Category fluency & $10.9(5.3)$ & $8.4(4.0)$ & $9.2(3.1)$ & $9.3(2.8)$ & -1.2 & -4.7 to 2.2 & 0.45 \\
\hline Switching category & $7.8(3.31)$ & $7.6(3.5)$ & $8.7(2.6)$ & $8.1(2.2)$ & 0.1 & -2.6 to 2.8 & 0.94 \\
\hline CWIT, inhibition & $11.0(1.9)$ & $9.7(2.3)$ & $10.0(3.7)$ & $10.3(2.9)$ & -0.94 & -2.2 to 0.3 & 0.13 \\
\hline CWIT, shifting & $8.4(3.6)$ & $8.7(3.7)$ & $9.3(8.8)$ & $10.8(1.8)$ & -1.4 & -3.7 to 0.8 & 0.18 \\
\hline CWIT, inhibition errors & $10.6(2.0)$ & $9.1(3.0)$ & $10.2(2.0)$ & $11.00(1.1)$ & -2.1 & -3.8 to -0.5 & 0.02 \\
\hline CWIT, shifting errors & $8.7(3.2)$ & $9.4(2.8)$ & $9.1(4.0)$ & $10.4(1.4)$ & -0.9 & -3.2 to 1.4 & 0.40 \\
\hline TMT, shifting & $7.0(4.4)$ & $5.7(4.2)$ & $9.0(1.5)$ & $9.3(3.7)$ & -1.5 & -4.8 to 1.7 & 0.33 \\
\hline TMT, shifting total errors & $9.9(2.2)$ & $8.3(3.9)$ & $11.0(1.1)$ & $9.8(3.2)$ & -0.2 & -3.8 to 3.5 & 0.93 \\
\hline \multicolumn{8}{|l|}{ Attention/working memory } \\
\hline PASAT, z-score & $43.9(8.8)$ & $42.9(9.3)$ & $36.9(8.7)$ & $41.6(10.7)$ & -4.4 & -13.0 to 4.1 & 0.29 \\
\hline \multicolumn{8}{|l|}{ Dichotic listening, T-score } \\
\hline Nonforced RE & $45.7(9.8)$ & $46.7(5.9)$ & $49.4(12.5)$ & $51.3(11.0)$ & -4.3 & -13.5 to 4.9 & 0.33 \\
\hline Nonforced LE & $43.6(6.0)$ & $44.3(5.1)$ & $49.9(8.4)$ & $44.8(7.7)$ & 2.0 & -5.7 to 9.6 & 0.59 \\
\hline Forced Rt RE & $43.6(6.0)$ & $44.3(5.1)$ & $49.9(8.4)$ & $56.8(11.5)$ & -5.8 & -15.8 to 4.3 & 0.24 \\
\hline Forced Rt LE & $43.3(7.0)$ & $45.3(6.6)$ & $48.7(10.8)$ & $41.4(7.4)$ & 4.5 & -3.6 to 12.6 & 0.25 \\
\hline Forced Lt RE & $48.6(10.1)$ & $52.6(8.0)$ & $46.8(8.2)$ & $47.8(8.3)$ & 3.7 & -2.0 to 9.5 & 0.19 \\
\hline Forced Lt LE & $44.9(9.2)$ & $46.0(9.1)$ & $53.9(7.6)$ & $50.6(7.6)$ & 2.4 & -3.8 to 8.7 & 0.42 \\
\hline Digit forward, scaled score & $11.0(2.34)$ & $10.8(2.4)$ & $11.8(2.8)$ & $12.1(2.9)$ & -0.8 & -2.9 to 1.2 & 0.40 \\
\hline Digit backward, scaled score & $9.4(4.3)$ & $6.7(2.6)$ & $9.0(2.1)$ & $8.5(2.3)$ & -2.0 & -4.3 to 0.3 & 0.08 \\
\hline \multicolumn{8}{|l|}{ Mood \& behavior } \\
\hline BAI, raw score & $9.3(4.9)$ & $4.9(3.8)$ & $10.3(7.2)$ & $9.4(6.50)$ & -3.3 & -8.9 to 2.2 & 0.23 \\
\hline BDI, raw score & $5.8(4.8)$ & $3.7(2.1)$ & $7.4(5.8)$ & $6.0(7.2)$ & -1.6 & -7.1 to 3.9 & 0.55 \\
\hline FrSBe, total score, T-score & $53.8(20.3)$ & $52.3(17.0)$ & $65.7(21.9)$ & $51.8(16.6)$ & 7.4 & -4.0 to 18.8 & 0.19 \\
\hline FrSBe, apathy, T-score & $56.4(20.1)$ & $53.1(13.7)$ & $68.0(23.5)$ & $53.5(14.8)$ & 4.7 & -6.3 to 15.7 & 0.38 \\
\hline FrSBe, disinhibition, T-score & $49.2(16.0)$ & $47.9(13.8)$ & $57.0(15.4)$ & $51.1(12.9)$ & 0.61 & -9.9 to 11.1 & 0.90 \\
\hline FrSBe, executive dysfunction, T-score & $52.8(14.2)$ & $53.7(14.9)$ & $60.7(18.0)$ & $49.0(13.5)$ & 9.2 & -1.3 to 19.6 & 0.08 \\
\hline
\end{tabular}

Diff = baseline - adjusted difference/intercept; LE = left ear; RE = right ear.

Values for preoperative and 6 months are given as mean (SD). Boldface type indicates statistical significance.

were reported after cZi DBS in PD. We did not observe the decline in verbal fluency that has been one of the most frequently reported cognitive outcomes after STN DBS and GPi DBS in PD. We did, however, notice a negative trend regarding both phonemic and category fluency at the 6- and 24-month follow-up, but neither reached statistical significance in this study. This could be due to a rather small sample size and might thus be a false-negative finding. Should this be a recurring finding in later studies, it would be an interesting and important difference between
STN DBS and cZi DBS that would warrant further investigations.

Because this study was part of a clinical trial, we did not complete a power analysis prior to the initiation of the data collection to determine the optimal sample sizes. We are, however, aware of the limited sample size in our material, especially regarding the DBS-BMT comparisons at 6 months. In the long-term follow-up we analyze a somewhat larger sample, because both groups have been merged at this point. A limited sample size will, of course, require 


\begin{tabular}{|c|c|c|c|c|c|}
\hline Test & Preop & 6 Mos & 24 Mos & $\begin{array}{l}\text { Preop vs } 6 \text { Mos } \\
\text { Adjusted } p \text { Value }\end{array}$ & $\begin{array}{l}\text { Preop vs } 24 \text { Mos } \\
\text { Adjusted } p \text { Value }\end{array}$ \\
\hline \multicolumn{6}{|l|}{ Global cognition } \\
\hline Ravens's Coloured Progressive Matrices (\%) & $60.4(45.1-75.6)$ & $67.2(50.5-83.9)$ & $52.0(38.7-65.3)$ & 0.558 & 0.181 \\
\hline \multicolumn{6}{|c|}{ Memory, T-score } \\
\hline Claeson-Dahls retention & $49.2(45.7-52.7)$ & $43.8(37.8-49.8)$ & $47.3(41.5-53.1)$ & 0.185 & 0.792 \\
\hline Claeson-Dahls verbal learning & $46.1(41.2-51.0)$ & $45.4(42.0-48.7)$ & $42.1(37.1-47.0)$ & 0.909 & 0.246 \\
\hline BVMT-R recall & $40.0(35.1-44.9)$ & $38.8(32.7-45.0)$ & $39.2(34.0-44.5)$ & 0.889 & 0.920 \\
\hline BVMT-R learning & $54.9(50.5-59.3)$ & $53.3(47.6-59.0)$ & $51.7(44.7-58.8)$ & 0.879 & 0.689 \\
\hline BVMT-R delayed recall & $47.1(42.0-52.3)$ & $45.3(39.4-51.3)$ & $45.8(39.8-51.8)$ & 0.827 & 0.899 \\
\hline \multicolumn{6}{|l|}{ Executive function, scaled score } \\
\hline Phonemic fluency & $10.72(9.12-12.3)$ & $9.74(8.45-11.0)$ & $9.41(7.89-10.9)$ & 0.173 & 0.129 \\
\hline Category fluency & $10.18(8.16-12.2)$ & $7.90(5.76-10.0)$ & $8.98(6.95-11.0)$ & 0.055 & 0.378 \\
\hline Switching category & $7.96(6.48-9.44)$ & $8.00(6.57-9.43)$ & $6.40(4.66-8.14)$ & 0.998 & 0.104 \\
\hline CWIT, inhibition & $10.58(9.30-11.9)$ & $9.29(7.60-11.0)$ & $8.81(6.71-10.9)$ & 0.058 & 0.084 \\
\hline CWIT, shifting & $9.57(7.86-11.3)$ & $8.94(6.83-11.0)$ & $8.29(5.96-10.6)$ & 0.728 & 0.383 \\
\hline CWIT, inhibition errors & $10.76(9.93-11.6)$ & $9.51(7.85-11.2)$ & $10.58(9.65-11.5)$ & 0.256 & 0.898 \\
\hline CWIT, shifting errors & $9.52(8.21-10.8)$ & $9.42(7.15-11.7)$ & $9.21(7.31-11.1)$ & 0.994 & 0.898 \\
\hline TMT, shifting & $8.22(6.21-10.24)$ & $6.65(4.73-8.57)$ & $7.10(4.97-9.23)$ & 0.055 & 0.338 \\
\hline TMT, shifting total errors & $9.91(8.62-11.2)$ & $8.71(7.08-10.3)$ & $9.82(8.32-11.3)$ & 0.090 & 0.977 \\
\hline \multicolumn{6}{|l|}{ Attention/working memory } \\
\hline PASAT, z-score & $43.0(36.6-49.4)$ & $40.2(35.1-45.2)$ & $34.7(28.3-41.0)$ & 0.337 & 0.011 \\
\hline \multicolumn{6}{|l|}{ Dichotic listening, T-score } \\
\hline Nonforced RE & $48.6(43.7-53.6)$ & $50.8(46.6-55.1)$ & $53.7(47.5-59.9)$ & 0.462 & 0.184 \\
\hline Nonforced LE & $43.9(40.3-47.5)$ & $44.5(41.9-47.1)$ & $43.1(40.7-45.5)$ & 0.938 & 0.871 \\
\hline Forced Rt RE & $50.6(44.5-56.8)$ & $51.3(46.4-56.3)$ & $51.7(45.3-58.2)$ & 0.938 & 0.918 \\
\hline Forced Rt LE & $42.3(38.7-45.9)$ & $42.9(39.7-46.0)$ & $45.5(41.9-49.2)$ & 0.952 & 0.300 \\
\hline Forced Lt RE & $48.1(43.6-52.7)$ & $50.5(46.0-54.9)$ & $52.6(48.5-56.7)$ & 0.475 & 0.049 \\
\hline Forced Lt LE & $47.7(43.5-51.9)$ & $47.7(43.5-51.9)$ & $46.6(43.3-49.8)$ & 1.000 & 0.739 \\
\hline Digit forward, scaled score & $11.7(10.24-13.1)$ & $11.4(10.0-12.8)$ & $10.3(8.83-11.7)$ & 0.860 & 0.068 \\
\hline Digit backward, scaled score & $9.08(7.19-10.97)$ & $7.92(6.38-9.47)$ & $7.66(6.17-9.15)$ & 0.392 & 0.236 \\
\hline \multicolumn{6}{|l|}{ Mood \& behavior } \\
\hline BAI, raw score & $9.44(6.48-12.41)$ & $4.76(2.95-6.57)$ & $7.53(3.42-11.65)$ & 0.004 & 0.660 \\
\hline BDI, raw score & $6.09(3.21-8.98)$ & $4.39(2.82-5.96)$ & $8.46(4.27-12.64)$ & 0.324 & 0.531 \\
\hline FrSBe, total score, T-score & $53.2(44.2-62.1)$ & $52.9(44.5-61.3)$ & 59.7 (50.7-68.7) & 0.994 & 0.090 \\
\hline FrSBe, apathy, T-score & $55.1(45.7-64.4)$ & $54.4(47.4-61.4)$ & $61.4(52.8-69.9)$ & 0.976 & 0.258 \\
\hline FrSBe, disinhibition, T-score & $50.2(43.1-57.3)$ & $50.1(42.6-57.7)$ & $52.3(45.5-59.2)$ & 0.999 & 0.606 \\
\hline FrSBe, executive dysfunction, T-score & $51.1(44.4-58.5)$ & $52.8(45.6-60.0)$ & $59.4(51.7-67.0)$ & 0.794 & 0.007 \\
\hline
\end{tabular}

Values for preoperative, 6 months, and 24 months are given as mean $(95 \% \mathrm{Cl})$. Boldface type indicates statistical significance.

that our results are confirmed in future studies, preferably with larger sample sizes. Because this is the first study to investigate the cognitive effects of cZi DBS in PD patients, we still believe that our results are of interest and that our study will encourage others to verify or dispute our findings.

Also, with repeated assessments there is a risk of practice/learning effects. To limit this, parallel versions of tests were used, where available. Nevertheless, the inclusion of a BMT group for comparison of the short-term cognitive and behavioral effects of cZi DBS, and the long-term follow-up of the patients at 24 months, are methodological advantages of this study. We did not conduct assessments with DBS on/off, which makes us unable to draw any conclusions as to whether the changes we see are due to stimulation or surgical intervention.

\section{Conclusions}

To our knowledge, this is the first randomized longterm follow-up study focusing on the cognitive effects of cZi DBS surgery in patients with PD. DBS in the cZi in 
patients with PD does not produce any global adverse effects on cognition, mood, or behavior. There were no differences in global cognitive status at 6 months between patients randomized to BMT and patients who underwent cZi DBS. At 6- and 24-month follow-up, cZi DBS showed no major changes in global cognitive functioning. However, cZi DBS was associated with a decline in attention and self-reported executive function at the 24-month followup. A transient improvement with regard to symptoms of anxiety during the first 6 months, when compared to the BMT group, was also found. In contrast, cZi DBS did not result in a decline of verbal fluency as has been consistently documented following STN DBS. We argue that the cZi as a target for DBS in PD is an addition to the established targets (STN, GPi, and Vim). Additional targets such as the cZi, with good effect on tremor and few cognitive side effects, can increase the options to modify surgical treatment for individual patients based on clinical profile and symptomatology.

\section{Acknowledgments}

We would like to thank Björn Häggström for some of the initial neuropsychological assessments in this sample and Per Liv for performing a substantial part of the statistical analyses.

\section{References}

1. Aarsland D, Brønnick K, Fladby T: Mild cognitive impairment in Parkinson's disease. Curr Neurol Neurosci Rep 11:371-378, 2011

2. Andy OJ, Jurko MF, Sias FR Jr: Subthalamotomy in treatment of Parkinsonian tremor. J Neurosurg 20:860-870, 1963

3. Beck AT, Steer RA: BAI-Beck Anxiety Inventory: manual, Svensk version. Stockholm: Pearson Assessment \& Information, 2009

4. Beck AT, Steer RA, Brown GK: BDI-II: Beck Depression Inventory: manual, Svensk version, ed 2. Stockholm: Psykologiförlaget, 2006

5. Benedict RHB: Brief Visuospatial Memory Test-Revised. Odessa, FL: Psychological Assessment Resources, Inc., 1997

6. Blomstedt P, Sandvik U, Fytagoridis A, Tisch S: The posterior subthalamic area in the treatment of movement disorders: past, present, and future. Neurosurgery 64:1029-1042, 2009

7. Blomstedt P, Stenmark Persson R, Hariz GM, Linder J, Fredricks A, Häggström $\mathrm{B}$, et al: Deep brain stimulation in the caudal zona incerta versus best medical treatment in patients with Parkinson's disease: a randomised blinded evaluation. J Neurol Neurosurg Psychiatry 89:710-716, 2018

8. Claeson LE, Esbjörnsson E, Tännérus BM, Wahlbin M: Claeson-Dahls test för inlärning och mine: manual, Reviderad version. Stockholm: Psykologiförlaget, 1998

9. Combs HL, Folley BS, Berry DT, Segerstrom SC, Han DY, Anderson-Mooney AJ, et al: Cognition and depression following deep brain stimulation of the subthalamic nucleus and globus pallidus pars internus in Parkinson's disease: a metaanalysis. Neuropsychol Rev 25:439-454, 2015

10. Delis DC, Kaplan E, Kramer JH: D-KEFS. Svenskt manualsupplement. Stockholm: Psykologiförlaget, 2005

11. Deuschl G, Raethjen J, Hellriegel H, Elble R: Treatment of patients with essential tremor. Lancet Neurol 10:148-161, 2011

12. Deuschl G, Schade-Brittinger C, Krack P, Volkmann J, Schäfer H, Bötzel K, et al: A randomized trial of deep-brain stimulation for Parkinson's disease. N Engl J Med 355:896908, 2006
13. Elgh E, Domellöf M, Linder J, Edström M, Stenlund H, Forsgren L: Cognitive function in early Parkinson's disease: a population-based study. Eur J Neurol 16:1278-1284, 2009

14. Fager CA: Evaluation of thalamic and subthalamic surgical lesions in the alleviation of Parkinson's disease. J Neurosurg 28:145-149, 1968

15. Foltynie T, Brayne CEG, Robbins TW, Barker RA: The cognitive ability of an incident cohort of Parkinson's patients in the UK. The CamPaIGN study. Brain 127:550-560, 2004

16. Foltynie T, Hariz MI: Surgical management of Parkinson's disease. Expert Rev Neurother 10:903-914, 2010

17. Gootjes L, Van Strien JW, Bouma A: Age effects in identifying and localising dichotic stimuli: a corpus callosum deficit? J Clin Exp Neuropsychol 26:826-837, 2004

18. Grace J, Malloy PF: Frontal Systems Behavior Scale (FrSBE): Professional Manual. Lutz, FL: Psychological Assessment Resources, Inc., 2001

19. Gratwicke J, Jahanshahi M, Foltynie T: Parkinson's disease dementia: a neural networks perspective. Brain 138:14541476, 2015

20. Heaton RK, Chelune GJ, Talley JL, Kay GG: Wisconsin Card Sorting Test Manual: Revised and Expanded. Lutz, FL: Psychological Assessment Resources, Inc., 1993

21. Hely MA, Reid WGJ, Adena MA, Halliday GM, Morris JGL: The Sydney multicenter study of Parkinson's disease: the inevitability of dementia at 20 years. Mov Disord 23:837-844, 2008

22. Houdart R, Mamo H, Dondey M, Cophignon J: [Results of subthalamic coagulations in Parkinson's disease (apropos of 50 cases).] Rev Neurol (Paris) 112:521-529, 1965 (French)

23. Hugdahl K, Asbjørnsen A: Dikotisk lyssning med CV-stavelser: manual. Stockholm: Psykologiförlaget, 1994

24. Hugdahl K, Westerhausen R, Alho K, Medvedev S, Laine M, Hämäläinen $\mathrm{H}$ : Attention and cognitive control: unfolding the dichotic listening story. Scand J Psychol 50:11-22, 2009

25. Jahanshahi M: Risky choices link the subthalamic nucleus with pathological gambling in Parkinson's disease. Mov Disord 28:1617-1619, 2013

26. Jahanshahi M, Dirnberger G, Fuller R, Frith CD: The role of the dorsolateral prefrontal cortex in random number generation: a study with positron emission tomography. Neuroimage 12:713-725, 2000

27. Jahanshahi M, Pote I, Torkamani M, Kefalopoulou ZM, Zrinzo L, Limousin P, et al: Deep brain stimulation of the subthalamic nucleus is associated with lower response thresholds when patients with Parkinson's disease act under speed pressure. Mov Disord 30:S341-S342, 2015

28. Litvan I, Goldman JG, Tröster AI, Schmand BA, Weintraub D: Mild cognitive impairment in de novo Parkinson's disease according to movement disorder guidelines. Mov Disord 27:1707, 2012

29. Mundinger F: [Subthalamotomy for the treatment of extrapyramidal movement disorders.] Dtsch Med Wochenschr 90:2002-2007, 1965 (German)

30. Muslimovic D, Post B, Speelman JD, Schmand B: Cognitive profile of patients with newly diagnosed Parkinson disease. Neurology 65:1239-1245, 2005

31. Okun MS, Foote KD: Parkinson's disease DBS: what, when, who and why? The time has come to tailor DBS targets. Expert Rev Neurother 10:1847-1857, 2010

32. Parsons TD, Rogers SA, Braaten AJ, Woods SP, Tröster AI: Cognitive sequelae of subthalamic nucleus deep brain stimulation in Parkinson's disease: a meta-analysis. Lancet Neurol 5:578-588, 2006

33. Plaha P, Ben-Shlomo Y, Patel NK, Gill SS: Stimulation of the caudal zona incerta is superior to stimulation of the subthalamic nucleus in improving contralateral parkinsonism. Brain 129:1732-1747, 2006

34. Raven J, Raven JC, Court JH: Manual for Raven's Progres- 
sive Matrices and Vocabulary Scales. Section 2: The Coloured Progressive Matrices. San Antonio, TX: Harcourt Assessment, Inc., 1998

35. Sjöberg RL, Häggström B, Philipsson J, Linder J, Hariz M, Blomstedt P: Laterality and deep brain stimulation of the subthalamic nucleus: applying a dichotic listening task to patients treated for Parkinson's disease. Neurocase 21:601606, 2015

36. Smeding HM, Speelman JD, Koning-Haanstra M, Schuurman PR, Nijssen P, van Laar T, et al: Neuropsychological effects of bilateral STN stimulation in Parkinson disease: a controlled study. Neurology 66:1830-1836, 2006

37. Story JL, French LA, Chou SN, Meier MJ: Experiences with subthalamic lesions in patients with movement disorders. Confin Neurol 26:218-221, 1965

38. Thobois S, Hotton GR, Pinto S, Wilkinson L, LimousinDowsey P, Brooks DJ, et al: STN stimulation alters pallidalfrontal coupling during response selection under competition. J Cereb Blood Flow Metab 27:1173-1184, 2007

39. Weaver FM, Follett K, Stern M, Hur K, Harris C, Marks WJ $\mathrm{Jr}$, et al: Bilateral deep brain stimulation vs best medical therapy for patients with advanced Parkinson disease: a randomized controlled trial. JAMA 301:63-73, 2009

40. Wechsler D: Wechsler Adult Intelligence Scale-Svensk version, ed 4. Stockholm: Pearson, Inc., 2010

41. Williams A, Gill S, Varma T, Jenkinson C, Quinn N, Mitchell R, et al: Deep brain stimulation plus best medical therapy versus best medical therapy alone for advanced Parkinson's disease (PD SURG trial): a randomised, open-label trial. Lancet Neurol 9:581-591, 2010

42. Witt K, Daniels C, Reiff J, Krack P, Volkmann J, Pinsker $\mathrm{MO}$, et al: Neuropsychological and psychiatric changes after deep brain stimulation for Parkinson's disease: a randomised, multicentre study. Lancet Neurol 7:605-614, 2008

43. Wu B, Han L, Sun BM, Hu XW, Wang XP: Influence of deep brain stimulation of the subthalamic nucleus on cognitive function in patients with Parkinson's disease. Neurosci Bull 30:153-161, 2014
44. York MK, Dulay M, Macias A, Levin HS, Grossman R, Simpson R, et al: Cognitive declines following bilateral subthalamic nucleus deep brain stimulation for the treatment of Parkinson's disease. J Neurol Neurosurg Psychiatry 79:789-795, 2008

\section{Disclosures}

Dr. Hariz has received honoraria and travel expenses from Medtronic and Boston Scientific for speaking at meetings. Dr. Blomstedt is a consultant for Abbott, Boston Scientific, and Medtronic, and is a shareholder in Mithridaticum AB. Ms. Philipson has received scholarships and grants to cover travel expenses for attending national and international conferences and international research stays from the Swedish Neuropsychological Society, Neurofonden, Parkinsonförbundet, and Cederbergsstiftelsen.

\section{Author Contributions}

Conception and design: Philipson, Jahanshahi. Acquisition of data: Philipson, Blomstedt, Fredricks, Hariz, Stenmark Persson. Analysis and interpretation of data: Philipson. Drafting the article: Philipson. Critically revising the article: Philipson, Blomstedt, Hariz, Stenmark Persson, Jahanshahi. Reviewed submitted version of manuscript: all authors. Approved the final version of the manuscript on behalf of all authors: Philipson. Statistical analysis: Philipson. Administrative/technical/material support: Blomstedt, Fredricks. Study supervision: Blomstedt, Hariz, Jahanshahi.

\section{Correspondence}

Johanna Philipson: Umeå University, Umeå, Sweden. johanna. philipsson@umu.se. 